\title{
USO FERTILIZANTE DE RESÍDUO DA INDÚSTRIA PROCESSADORA DE GOIABAS ${ }^{1}$
}

\author{
JOSÉ RICARDO MANTOVANI², MÁRCIO CLEBER DE MEDEIROS CORRÊA³, MARA CRISTINA PESSÔA DA CRUZ4, \\ MANOEL EVARISTO FERREIRA ${ }^{5}$, WILLIAM NATALE ${ }^{6}$
}

\begin{abstract}
RESUMO - O resíduo da indústria processadora de goiabas vem sendo aplicado em algumas áreas, entretanto não é conhecido o seu efeito na fertilidade do solo. Foi realizado experimento em casa de vegetação, com o objetivo de avaliar o efeito da aplicação do resíduo da indústria processadora de goiabas na fertilidade do solo, tendo como planta-teste o milho. O delineamento experimental utilizado foi em blocos ao acaso, em esquema fatorial 5x2, com quatro repetições, combinando-se cinco doses do resíduo da indústria processadora de goiabas $\left(0 ; 20 ; 40 ; 60\right.$ e $\left.80 \mathrm{t} \mathrm{ha}{ }^{-1}\right)$ na presença e ausência de adubação mineral. A adição de resíduo da indústria processadora de goiabas propiciou aumento nos teores de $\mathrm{P}$ e $\mathrm{K}$ do solo. O resíduo da indústria processadora de goiabas é uma fonte de liberação lenta de $\mathrm{N}$ e $\mathrm{P}$ para as plantas.
\end{abstract}

Termos para indexação: Psidium guajava, adubação orgânica, adubação mineral, macronutrientes, micronutrientes, milho.

\section{FERTILIZER USE OF GUAVA PROCESSING INDUSTRY WASTE}

\begin{abstract}
The guava processing industry waste (GPIW) has been used in some areas, however is not known its effect on soil fertility. A greenhouse experiment was carried out to evaluate the effect of GPIW on soil fertility, using corn as testing plant. The experiment was in randomized blocks design combining five GPIW rates $\left(0 ; 20 ; 40 ; 60\right.$ and $\left.80 \mathrm{tha}^{-1}\right)$ in the absence and the presence of mineral fertilization. The GPIW increased P and $\mathrm{K}$ concentration and behaved as a slow release source of $\mathrm{N}$ and $\mathrm{P}$ for corn.
\end{abstract}

Index Terms: Psidium guajava, organic manure, mineral fertilizer, macronutrients, micronutrients, corn.

\section{INTRODUÇÃO}

O Brasil é um dos maiores produtores de goiaba do mundo, com volume estimado, em 2002, de cerca de 390 mil t. A produção concentra-se nas regiões Sudeste e Nordeste do País, sendo o Estado de São Paulo responsável por mais de $60 \%$ do volume nacional da fruta (Agrianual, 2004).

Além do consumo in natura, produtos industrializados, como goiabada, geléia e suco, são a principal forma de consumo da fruta no Brasil. No processamento da goiaba, após o despolpamento e a lavagem com água clorada, obtém-se um resíduo composto principalmente por sementes, na proporção de 4 a $12 \%$ da massa total dos frutos beneficiados. Esse resíduo apresenta, de acordo com Fernandes et al. (2002), 17; 2 e $3 \mathrm{~g}$ $\mathrm{kg}^{-1}$ de N, P e K, respectivamente. Dessa maneira, a aplicação de $40 \mathrm{t} \mathrm{ha}^{-1}$ do resíduo (base seca) adicionaria ao solo as quantidades totais de 680; 80 e $120 \mathrm{~kg} \mathrm{ha}^{-1}$ de N, Pe K, respectivamente. Esses valores correspondem, respectivamente, a 5,7; 2,7 e 1,6 vezes as quantidades de N, P e K recomendadas por Santos \& Quaggio (1997) para aplicação anual em pomares de goiabeira com produtividade esperada de 30 a $40 \mathrm{t} \mathrm{ha}^{-1} \mathrm{de}$ frutos, em solos com teores médios de $\mathrm{P}$ e K.

Considerando que, no Estado de São Paulo, são produzidas 230 mil t ano ${ }^{-1}$ de frutas (Agrianual, 2004) e que 55\% desse volume é destinado à industrialização (130 mil t), considerando, ainda, que em média $8 \%$ da goiaba processada constitui o resíduo, ter-se-ia, anualmente, produção de 10 mil t do resíduo, nos quais estariam contidos, segundo Fernandes et al. (2002), cerca de 170 t de N, 20 t de P e 30 t de K, nutrientes que não estão sendo aproveitados.

Na literatura, são poucas as informações a respeito dos benefícios da aplicação do resíduo da indústria processadora de goiabas na fertilidade do solo. Em estudos feitos no Egito, ficou demonstrado, ainda, de forma incipiente, que a adição do resíduo aumentou os teores de nitrogênio total, fósforo disponível e potássio trocável do solo (El-Leboudi et al., 1988; Abd El-Moez, 1996a,b). Fernandes et al. (2002), em experimento em condições de laboratório, utilizaram o resíduo da indústria processadora de goiabas moído, como pó fino, e verificaram, ao final de 90 dias de incubação do resíduo com um Argissolo, aumento nos teores de matéria orgânica e de potássio do solo e decréscimo no valor de $\mathrm{pH}$.
No Brasil, o resíduo tem sido descartado pelas indústrias a céu aberto ou, raramente, em aterros sanitários, e, com isso, grande quantidade de nutrientes, que poderiam ser reciclados, não são aproveitados. Entretanto, algumas indústrias vêm aplicando esse material em seus pomares de goiabeiras ou em áreas destinadas a culturas anuais, como o milho. Todavia, tal utilização não segue critérios técnicos, o que se deve especialmente ao desconhecimento do seu efeito na fertilidade do solo. Dessa maneira, este trabalho teve como objetivo avaliar o efeito da aplicação do resíduo da indústria processadora de goiabas na fertilidade do solo.

\section{MATERIALEMÉTODOS}

O experimento foi realizado em casa de vegetação, no período de novembro de 2002 a março de 2003. Utilizou-se solo da camada arável ( 0 a $20 \mathrm{~cm}$ ) de um Latossolo Vermelho eutrófico, coletado no município de Borborema-SP, com a seguinte caracterização química de rotina (Raij et al., 1987): $\mathrm{P}$ resina $=15 \mathrm{mg} \mathrm{dm}{ }^{-3} ;$ M.O. $=29 \mathrm{~g} \mathrm{dm}^{-3} ; \mathrm{pH} \mathrm{em} \mathrm{CaCl}_{2} 0,01 \mathrm{~mol} \mathrm{~L}^{-1}=$ 5,$5 ; \mathrm{K}^{+}, \mathrm{Ca}^{2+}, \mathrm{Mg}^{2+}, \mathrm{H}+\mathrm{Al}, \mathrm{SB}$ e CTC, respectivamente, iguais a 2,3; 22; 13 ; 20;37 e $57 \mathrm{mmol}_{\mathrm{c}} \mathrm{dm}^{-3} ; \mathrm{V}=65 \%$; e granulométrica (Camargo et al., 1986): argila $=250 \mathrm{~g} \mathrm{~kg}^{-1} \mathrm{e}$ areia $=680 \mathrm{~g} \mathrm{~kg}^{-1}$. No solo, também foram feitas análises de $\mathrm{S}_{-} \mathrm{SO}_{4}^{2-}$ (Cantarella \& Prochnow, 2001), de B (Abreu et al., 1994) e de $\mathrm{Cu}, \mathrm{Fe}, \mathrm{Mn}$ e $\mathrm{Zn}$ (Abreu et al., 2001), cujos resultados foram, respectivamente, iguais a $7 ; 0,16 ; 0,8 ; 22 ; 24,8$ e $0,8 \mathrm{mg} \mathrm{dm}^{-3}$.

$\mathrm{O}$ resíduo da indústria processadora de goiabas foi seco em estufa acerca de $65^{\circ} \mathrm{C}$, analisado conforme descrito em Kiehl (1985), e os resultados obtidos foram os seguintes (base seca): $\mathrm{C}=355 \mathrm{~g} \mathrm{~kg}^{-1} ; \mathrm{N}=16$ $\mathrm{g} \mathrm{kg}^{-1}$; relação $\mathrm{C} / \mathrm{N}=22 / 1, \mathrm{P}, \mathrm{K}, \mathrm{Ca}$ e $\mathrm{Mg}$, respectivamente iguais a 2; 3; 0,5 e $0,5 \mathrm{~g} \mathrm{~kg}^{-1}$. Apresentou, ainda, 9; 39; 8 e $18 \mathrm{mg} \mathrm{kg}^{-1} \mathrm{de} \mathrm{Cu}, \mathrm{Fe}, \mathrm{Mn}$ e Zn, respectivamente.

Realizou-se um experimento para avaliar esse material como fertilizante. Para isso, empregou-se esquema fatorial $5 \times 2$, em delineamento em blocos ao acaso, com quatro repetições. Os tratamentos consistiram na combinação de cinco doses de resíduo da indústria processadora de goiabas, equivalentes a $0 ; 20 ; 40 ; 60$ e $80 \mathrm{t} \mathrm{ha}^{-1}$ (base seca), combinadas com presença e ausência de adubação mineral. O cálculo das doses do resíduo foi feito com base no volume de solo empregado, considerando a sua incorporação na camada de 0 a $20 \mathrm{~cm}$. A adubação mineral

\footnotetext{
${ }^{1}$ (Trabalho 026/2004). Recebido: 11/03/2004. Aceito para publicação: 05/08/2004.

${ }^{2}$ Aluno de doutorado do curso de Pós-Graduação em Agronomia (Produção Vegetal), FCAV/UNESP. Via de acesso Prof. Paulo Donato Castellane, s/n. CEP 14884900. Jaboticabal-SP. E-mail: mantovanijr@yahoo.com. Bolsista da FAPESP.

${ }^{3}$ Aluno de doutorado do curso de Pós-Graduação em Agronomia (Produção Vegetal), FCAV/UNESP. E-mail: mcleber@ fcav.unesp.br. Bolsista da FAPESP.

${ }^{4}$ Professora Assistente-Doutora do Departamento de Solos e Adubos, FCAV/UNESP. E-mail: mcpcruz@fcav.unesp.br

${ }^{5}$ Professor Titular do Departamento de Solos e Adubos, FCAV/UNESP. E-mail: evaristo@fcav.unesp.br

${ }^{6}$ Professor Adjunto do Departamento de Solos e Adubos, FCAV/UNESP. E-mail: natale@ fcav.unesp.br
} 

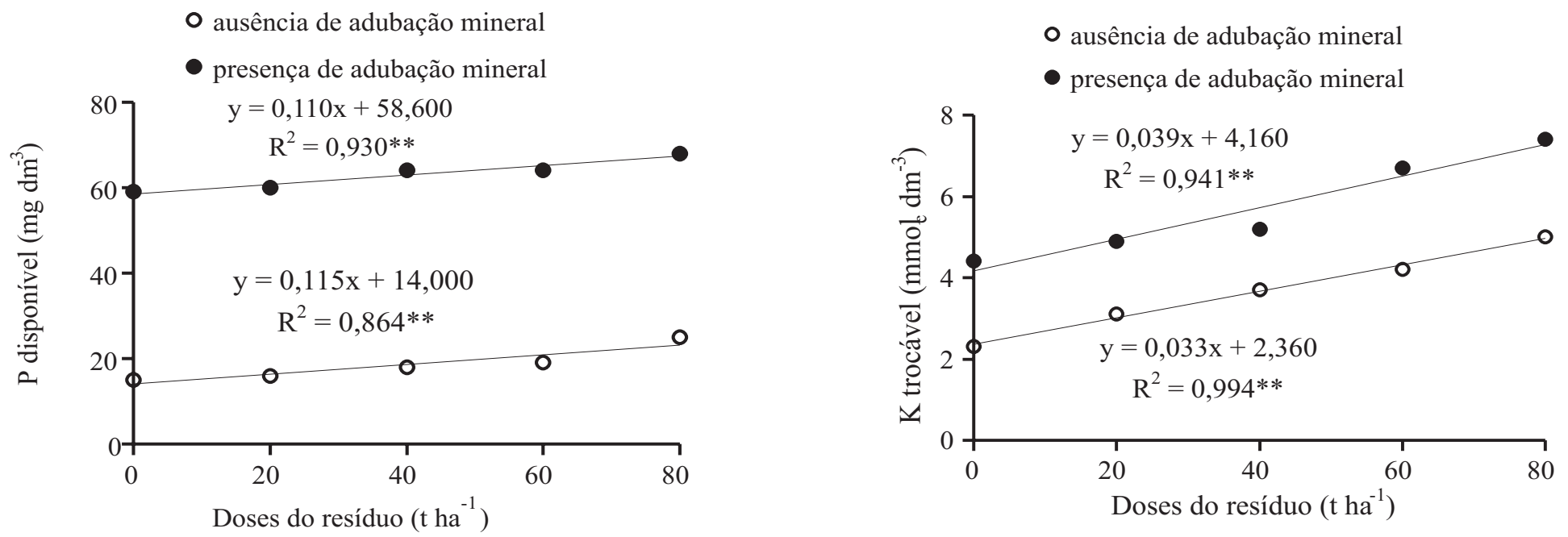

FIGURA 1 - Efeito do resíduo da indústria processadora de goiabas, na ausência e na presença de adubação mineral, nos teores de P e K do solo.

correspondeu, em mg dm ${ }^{-3}$, a 300 de N; 80 de P; 250 de K; 20 de S, e 1 de Zn.

O resíduo foi misturado, a seco, a porções de $5,8 \mathrm{dm}^{3}$ de solo, de acordo com cada tratamento, que foram transferidas para vasos de plástico com capacidade para 6 L. Em seguida, o solo foi umedecido com água desionizada a $70 \%$ da capacidade de retenção e submetido a incubação por 45 dias. Aos 20 dias de incubação, foi feita a adubação mineral, aplicando-se $100 \%$ da dose de $\mathrm{P}, \mathrm{S}$ e Zn, e $30 \%$ do $\mathrm{N}$ e do K. Foram usadas soluções preparadas a partir dos sais (p.a.): $\left(\mathrm{NH}_{4}\right)_{2} \mathrm{SO}_{4} ; \mathrm{NH}_{4} \mathrm{NO}_{3} ; \mathrm{KH}_{2} \mathrm{PO}_{4}$, e $\mathrm{ZnSO}_{4} .7 \mathrm{H}_{2} \mathrm{O}$. Ao término da incubação, o solo foi seco ao ar e amostrado para caracterização química (Raij et al., 1987). Após a amostragem, porções de $5,3 \mathrm{dm}^{3}$ de solo foram devolvidas para os vasos e reumedecidas a $70 \%$ da capacidade de retenção de água. Em seguida, semeou-se o milho (Zea mays L.), híbrido simples 8480 Dow AgroSciences, deixando-se, após o desbaste, cinco plantas por vaso. Foram feitas duas adubações em cobertura nos tratamentos com adubação mineral, aos 19 e aos 30 dias após a semeadura, aplicando-se, em cada uma delas, $35 \%$ da dose de $\mathrm{Ne}$ de $\mathrm{K}$, como $\mathrm{NH}_{4} \mathrm{NO}_{3}$ e $\mathrm{KNO}_{3}$. Durante a condução do experimento, a água foi reposta por meio de pesagens, duas a três vezes ao dia, e as quantidades variaram de acordo com o estágio das plantas e com a temperatura.

A colheita foi feita 41 dias após a semeadura, cortando-se as plantas rente à superfície do solo de cada vaso. A parte aérea das plantas foi lavada, seca em estufa acerca de $65^{\circ} \mathrm{C}$ até massa constante, moída e submetida a digestão nítrico-perclórica (Bataglia et al., 1983). Nos extratos, determinaram-se os teores de $\mathrm{P}$ por colorimetria, de $\mathrm{K}$ por fotometria de chama e de $\mathrm{Ca}, \mathrm{Mg}, \mathrm{Cu}, \mathrm{Fe}, \mathrm{Mn}$ e $\mathrm{Zn}$ por espectrofotometria de absorção atômica. Para a determinação do $\mathrm{N}$ total nas plantas, foram feitas digestão sulfúrica, destilação dos extratos em microdestilador Kjeldahl e subseqüente titulação do destilado (Sarruge \& Haag, 1974).

Os resultados obtidos foram submetidos a análises de variância e de regressão polinomial.

\section{RESULTADOS E DISCUSSÃO}

Verificou-se, após 45 dias de incubação, que a adição do resíduo da indústria processadora de goiabas afetou os teores de $\mathrm{P}$ disponível e de $\mathrm{K}$ trocável do solo, e a adubação mineral, por sua vez, afetou o pH e os teores de $\mathrm{P}, \mathrm{K}$ e H+Al. Os teores de M.O., Ca e Mg trocáveis e H+Al, e os valores de $\mathrm{pH}, \mathrm{SB}$ e V\% não foram alterados com a adição do resíduo. No caso do $\mathrm{Ca}$ e do $\mathrm{Mg}$, isso se deve aos baixos teores dos nutrientes no

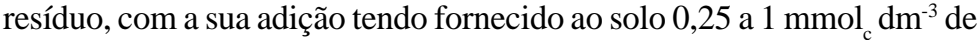
Ca e 0,42 a 1,68 $\mathrm{mmol}_{\mathrm{c}} \mathrm{dm}^{-3} \mathrm{de} \mathrm{Mg}$, o que é praticamente desprezível em relação aos teores iniciais do solo.

Os teores de $\mathrm{P}$ e de $\mathrm{K}$ do solo aumentaram linearmente com as doses do resíduo, tanto na ausência quanto na presença do adubo mineral (Figura 1). Na ausência da adubação mineral, os teores de $\mathrm{K}$ do solo, estimados pela equação de regressão, variaram de $2,4 \mathrm{mmol}_{\mathrm{c}} \mathrm{dm}^{-3}$, no tratamento-testemunha, para $5,0 \mathrm{mmol}_{\mathrm{c}} \mathrm{dm}^{-3}$ no tratamento que recebeu 80 t ha ${ }^{-1}$ do resíduo. Com a adubação mineral, os valores de $\mathrm{K}$ passaram de 4,2 para 7,3. Em relação ao $\mathrm{P}$, os teores no solo passaram de 14 para $23 \mathrm{mg} \mathrm{dm}$
${ }^{3}$ na ausência de adubação mineral e, na sua presença, os teores variaram de 59 para $67 \mathrm{mg} \mathrm{dm}^{-3}$, quando se comparou o tratamento-testemunha ao que recebeu a maior dose do resíduo. Portanto, os maiores aumentos no solo foram observados proporcionalmente para potássio. Fernandes et al. (2002) também constataram aumento linear nos teores de K do solo com a aplicação de até 87,6 tha $^{-1}$ de resíduo da indústria processadora de goiabas, na base seca, após 90 dias de incubação com o solo. Em relação ao P, os autores não verificaram alteração significativa no teor do nutriente no solo, com a adição do resíduo.

Considerando que, com a aplicação do equivalente a $80 \mathrm{t} \mathrm{ha}^{-1}$ do

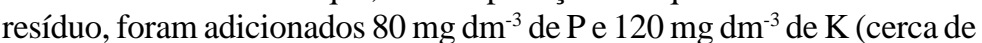
$3,1 \mathrm{mmol}_{\mathrm{c}} \mathrm{dm}^{-3}$ ), a recuperação foi de $12 \%$ e $11 \%$ para o P e de $86 \%$ e $100 \%$ para o $\mathrm{K}$ nos tratamentos sem e com adubação mineral, respectivamente, indicando que o $\mathrm{K}$ presente no resíduo, como esperado, é facilmente disponibilizado. Segundo Malavolta et al. (1989), o K é freqüentemente o nutriente mais prontamente liberado pelos resíduos orgânicos, por ser um elemento presente nos tecidos vegetais na forma iônica, não fazendo parte de compostos existentes nas plantas. De acordo com Cantarella et al. (1992), a liberação de P contido em materiais orgânicos, em geral, ocorre de forma relativamente lenta. Entretanto, há relatos de grandes acréscimos de $\mathrm{P}$ disponível em solos em períodos relativamente curtos, de 30 a 60 dias, com a adição de resíduos, como o composto de lixo urbano (Mazur et al., 1983; Abreu Junior et al., 2002).

$\mathrm{Na}$ ausência de adubação mineral, a matéria seca da parte aérea do milho aumentou linearmente com as doses do resíduo e, na presença, não houve variação significativa, ficando a produção em torno de $80 \mathrm{~g} /$ vaso (Figura 2). A produção média de matéria seca na presença de adubação mineral foi cerca de quatro vezes maior do que a obtida na ausência, no tratamento em que se aplicou a maior dose do resíduo.

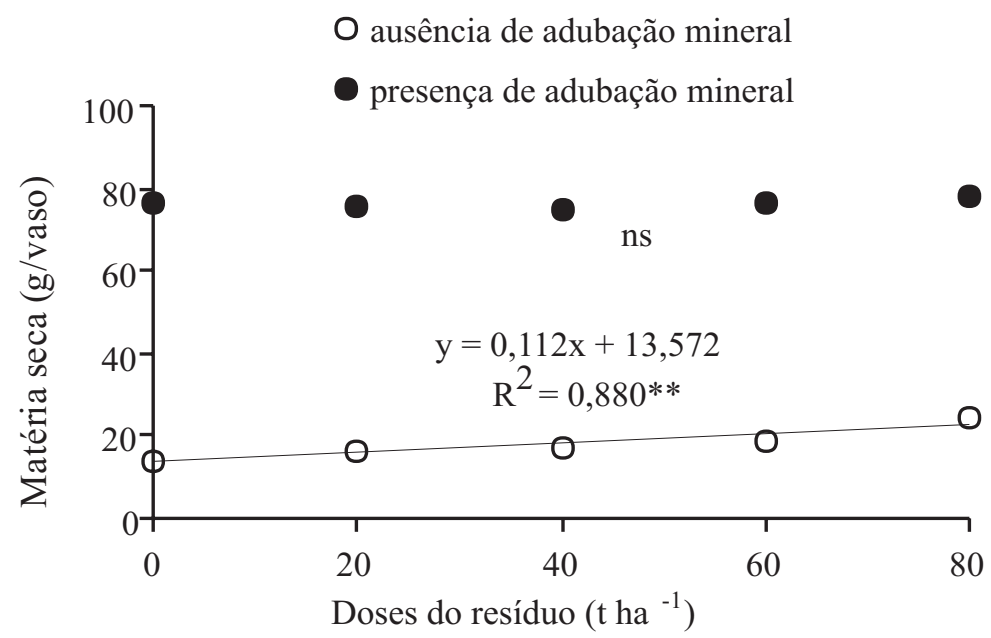

FIGURA 2 - Efeito do resíduo da indústria processadora de goiabas, na ausência e na presença de adubação mineral, na produção de matéria seca da parte aérea do milho. 
Com o emprego exclusivo do resíduo, além de limitação do crescimento das plantas, foram observados sintomas visuais de deficiência, particularmente de $\mathrm{P}$ e de $\mathrm{N}$, independentemente da dose. Os teores de $\mathrm{N}$ na parte aérea do milho variaram de 6,4 a $16,3 \mathrm{~g} \mathrm{~kg}^{-1} \mathrm{na}$ ausência de adubação mineral e de 12,3 a $15,0 \mathrm{~g} \mathrm{~kg}^{-1}$ na sua presença. Quanto ao P, os teores no milho variaram de 1,0 a 1,4 $\mathrm{g} \mathrm{kg}^{-1}$ e de 1,2 a 1,5 $\mathrm{g} \mathrm{kg}^{-1}$, nos tratamentos sem e com adubo mineral, respectivamente. Esses teores de $\mathrm{N}$ e de $\mathrm{P}$ são baixos, e uma explicação para isso é o efeito diluição, somado à baixa mineralização do $\mathrm{N}$ orgânico presente no resíduo. Com a aplicação do resíduo, foram adicionados 0; 160; 320; 480 e 640 mg $\mathrm{dm}^{-3}$ de N. No tratamento com adubação mineral, foram aplicados mais $300 \mathrm{mg} \mathrm{dm}^{-3} \mathrm{de} \mathrm{N}$. Assim, o crescimento do milho ou foi muito limitado pelo $\mathrm{P}$, ou o $\mathrm{N}$ do resíduo foi muito pouco mineralizado no intervalo de tempo avaliado, ou ambas as coisas ocorreram simultaneamente. Abd El-Moez (1996b) verificou que a aplicação de resíduo de sementes de goiabas promoveu o aumento da massa seca de Vicia faba e que, de modo geral, maiores rendimentos foram obtidos nos tratamentos que receberam resíduo mais adubação NPK, quando comparados àqueles que receberam apenas o resíduo ou a adubação mineral.

Houve aumento das quantidades acumuladas de $\mathrm{P}$ e de $\mathrm{K}$ na parte aérea do milho com a aplicação do resíduo, tanto na ausência como na presença de adubação mineral, tendo sido observado maior incremento de K (Figura 3). Quanto ao N, verificou-se, na presença de adubação mineral, diminuição linear das quantidades acumuladas do nutriente na parte aérea do milho com as doses do resíduo e, na ausência, houve efeito quadrático, com decréscimo das quantidades acumuladas de $\mathrm{N}$ pelo milho até a dose estimada de $46 \mathrm{t} \mathrm{ha}^{-1}$ do resíduo (Figura 3). Houve diminuição de $17 \%$ e $21 \%$ nas quantidades acumuladas de N na parte aérea do milho, na ausência e na presença de adubação mineral, respectivamente, quando se comparou o tratamento-testemunha com o que recebeu a maior dose do resíduo $\left(80 \mathrm{t} \mathrm{ha}^{-1}\right)$. Os resultados obtidos para $\mathrm{N}$ evidenciam, de certa maneira, a ocorrência de imobilização do $\mathrm{N}$ no solo com a adição do resíduo da indústria processadora de goiabas, mesmo esse material tendo uma relação $\mathrm{C} / \mathrm{N}$ inferior a 30:1. Considerando que não houve aumento no teor de matéria orgânica do solo com a adição do resíduo, que as sementes do resíduo permaneceram visíveis no solo ao final do experimento e que as plantas não conseguiram aproveitar o $\mathrm{N}$ do resíduo, pode-se inferir que a mineralização é lenta. Por outro lado, a liberação gradativa de $\mathrm{N}$ e $\mathrm{P}$ do resíduo pode trazer vantagens no caso da aplicação em culturas perenes, por diminuir as perdas de nitrogênio, devido à sua dinâmica no solo, e a fixação do fósforo, devido às características do solo.

As quantidades acumuladas de Mn e de Zn na parte aérea do milho aumentaram com a adição do resíduo, na ausência de adubação mineral, com as equações de regressão obtidas para $\mathrm{Mn}$ e $\mathrm{Zn}$, sendo, respectivamente, iguais a: $\mathrm{y}=10,767 \mathrm{x}+819,000 ; \mathrm{R}^{2}=0,939^{* *}, \mathrm{e} \quad \mathrm{y}=$ $0,039 x^{2}-2,248 x+264,814 ; R^{2}=0,971 * *$, em que y é a quantidade do nutriente (Mn ou $\mathrm{Zn}$ ) em $\mu \mathrm{g} /$ vaso e x é a quantidade de resíduo aplicado em $t$ ha $^{-1}$. Com a adubação mineral, os valores praticamente não se alteraram com as doses do resíduo, ficando em torno de $6.300 \mu \mathrm{g} / \mathrm{vaso}$ para $\mathrm{Mn}$, e $1.030 \mu \mathrm{g} / \mathrm{vaso}$ para $\mathrm{Zn}$.

As quantidades acumuladas de $\mathrm{Ca}, \mathrm{Mg}, \mathrm{Cu}$ e $\mathrm{Fe}$ na parte aérea do milho não foram alteradas pela adição do resíduo da indústria processadora de goiabas, tanto na ausência quanto na presença de adubação mineral.
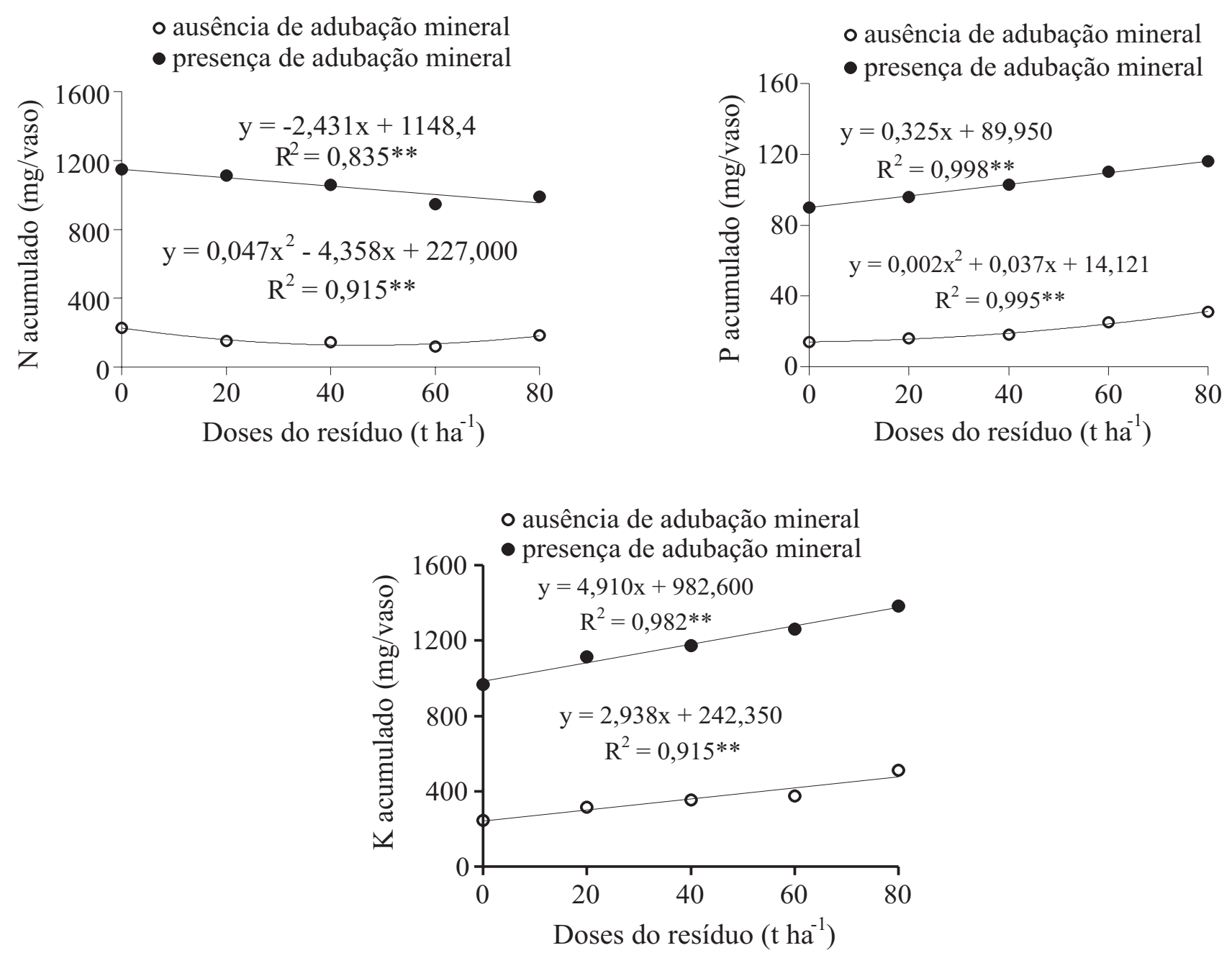

FIGURA 3 - Efeito do resíduo da indústria processadora de goiabas, na ausência e na presença de adubação mineral, nas quantidades acumuladas de $\mathrm{N}, \mathrm{P}$ e K na parte aérea do milho. 


\section{CONCLUSÕES}

1) A adição de resíduo da indústria processadora de goiabas propiciou aumento nos teores de $\mathrm{P}$ e $\mathrm{K}$ do solo.

2) O resíduo da indústria processadora de goiabas é uma fonte lenta de fornecimento de $\mathrm{N}$ e $\mathrm{P}$ para as plantas.

\section{REFERÊNCIASBIBLIOGRÁFICAS}

ABD EL-MOEZ, M.R. Dry matter yield and nutrient uptake of corn as affected by some organic wastes applied to a sandy soil. Annals of Agricultural Science, Cairo, v.34, p.1319-1330, 1996a.

ABD EL-MOEZ, M.R. Response of Vicia faba followed by Zea mays to application of mineral fertilizers and organic industrial waste. Annals of Agricultural Science, Cairo, v.34, p.1331-1343, $1996 \mathrm{~b}$.

ABREU, C.A.; ABREU, M.F.; RAIJ, B. van; BATAGLIA, O.C.; ANDRADE, J.C. Extraction of boron from soil by microware heating for ICP-AES determination. Communications in Soil Science and Plant Analysis, New York, v.25, p.3321-3333, 1994.

ABREU, C.A.; ABREU, M.F.; ANDRADE, J.C. Determinação de cobre, ferro, manganês, zinco, cádmio, cromo, níquel e chumbo em solos usando a solução de DTPA em pH 7,3. In: RAIJ, B. van; Andrade, J.C.; Cantarella, H.; Quaggio, J.A. (Eds.). Análise química para avaliação da fertilidade de solos tropicais. Campinas:Instituto Agronômico, 2001.p.240-250.

ABREU JUNIOR, C.H.; MURAOKA, T.; OLIVEIRA, F.C. Carbono, nitrogênio, fósforo e enxofre em solos tratados com composto de lixo urbano. Revista Brasileira de Ciência do Solo, Viçosa, v.26, p.769-780, 2002.

AGRIANUAL 2003: anuário da agricultura brasileira. São Paulo: FNP Consultoria \& Comércio, 2004. p.314-318.

BATAGLIA, O.C.; FURLANI, A.M.C.; TEIXEIRA, J.P.F.; FURLANI, P.R.; GALLO, J.R. Métodos de análise química de plantas. Campinas: Instituto Agronômico, 1983. 48p. (Boletim Técnico, 78).

CAMARGO, O.A.; MONIZ, A.C.; JORGE, J.A.; VALADARES, J.M.A.S. Métodos de análise química, mineralógica e física de solos do Instituto Agronômico de Campinas. Campinas: Instituto Agronômico, 1986. 94p. (Boletim Técnico, 106).
CANTARELLA, H.; ABREU, C.A.A.; BERTON, R.S. Fornecimento de nutrientes pela matéria orgânica do solo. In: GUERRINI, I.A.; BÜLL, L.T. (Ed.). ENCONTRO SOBRE MATÉRIA ORGÂNICADO SOLO: PROBLEMAS E SOLUÇÕES, 1992, Botucatu, Anais... Botucatu: Faculdade de Ciências Agronômicas, 1992. p.63-122.

CANTARELLA, H.; PROCHNOW, L.I. Determinação de sulfato em solos. In: RAIJ, B. van; Andrade, J.C.; Cantarella, H.; Quaggio, J.A. (Ed.). Análise química para avaliação da fertilidade de solos tropicais. Campinas:Instituto Agronômico, 2001. p.225-230.

EL-LEBOUDI, A.E.; IBRAHIM, S.A.; ABD EL-MOEZ, M.R. A trial for getting benefit from organic wastes of food industry. I. Effect on soil properties. Egypt Journal of Soil Science, Cairo, v.28, p.289298, 1988.

FERNANDES, G.C.; CORRÊA, M.C.M.; PRADO, R.M.; NATALE, W.; SILVA, M.A.C. Uso Agronômico do resíduo da indústria processadora de goiaba. In: CONGRESSO BRASILEIRO DE OLERICULTURA,42.; CONGRESSO LATINO-AMERICANO DE HORTICULTURA,11., 2002. Uberlândia. Resumos... Brasília: Horticultura Brasileira, 2002. Suplemento 2. CD-ROM.

KIEHL, E.J. Fertilizantes orgânicos. São Paulo:Agronômica Ceres, 1985. 492p.

MALAVOLTA, E.; VITTI, G.C.; OLIVEIRA, S.A. Avaliação do estado nutricional das plantas. Piracicaba: Associação Brasileira para Pesquisa da Potassa e do Fosfato, 1989. 201p.

MAZUR, N.; SANTOS, G.A.; VELLOSO, A.C.X. Efeito do composto de resíduo urbano na disponibilidade de fósforo em solo ácido. Revista Brasileira de Ciência do Solo, Campinas, v.7, p.153-156, 1983.

RAIJ, B. van; QUAGGIO, J.A.; CANTARELLA, H.; FERREIRA, M.E.; LOPES, A.S., BATAGLIA, O.C. Análise química do solo para fins de fertilidade. Campinas: Fundação Cargill, 1987. 170p.

SANTOS, R.R.; QUAGGIO, J.A. Goiaba. In: RAIJ, B. van; CANTARELLA, H.; QUAGGIO, J.A.; FURLANI, A.M.C. (Ed.) Recomendação de adubação e calagem para o estado de São Paulo. Campinas:Instituto Agronômico, 1997. p.143-144. (Boletim Técnico, 100).

SARRUGE, J.R.; HAAG, H.P. Análises químicas em plantas. Piracicaba:ESALQ, 1974. 56p. 